\title{
Editorial
}

\section{Intra-alveolar haemorrhage in sudden infant death syndrome: a cause for concern?}

It is generally accepted that an unknown proportion of cot deaths are a result of suffocation. Accidental suffocation or "overlaying" still remains a controversial issue which offends the political correctness of the co-sleeping lobby. Secretly recorded videos have removed all remaining doubts that occasional parents smother their children deliberately. The work of Meadow, Southall and others has taught us the type of people who repeatedly smother their babies, how they do it, and how to recognise them. ${ }^{12}$ One reason for any remaining disagreement is the lack of consistent objective pathological markers of asphyxia in infants. In this issue ( $\mathrm{p}$ 581) Yukawa et al report their results using digital image analysis to quantitate alveolar haemorrhage in a series of sudden unexpected infant deaths (SUDI).

Alveolar haemorrhage in early childhood has many causes. In the neonatal period haemorrhagic pulmonary oedema is common ${ }^{3}$ and causes include birth asphyxia, sepsis, heart failure, fluid overload, hypothermia, haemostatic failure, oxygen toxicity, and inborn errors of metabolism. In older babies pulmonary haemorrhage is less common, and causes such as heart failure, trauma, bleeding diatheses, idiopathic pulmonary haemosiderosis, antibasement-membrane antibodies, milk allergy, aspiration, vascular malformations, bronchiectasis, and other localised conditions account for occasional cases. An outbreak of pulmonary haemorrhage in the USA was attributed to environmental contamination by a toxigenic fungus. ${ }^{4}$ Until recently, pink froth in the respiratory tract and alveolar haemorrhage were accepted as a non-discriminatory finding in SIDS victims. ${ }^{5}$ However, those who work in the field have long associated marked haemorrhage in the respiratory tract with mechanical asphyxia, but systematic studies to back this up were lacking.

Southall reported that bleeding from the nose and mouth was seen in 11 of 38 patients undergoing covert video surveillance for suspicion of induced illness, but in none of 46 control children with apparent life threatening events from natural medical causes. ${ }^{2}$ It is not known if the blood in these cases of near suffocation results from local trauma or bleeding from the lower respiratory tract. Shadowing on chest $x$ rays after such events may well be due to pulmonary haemorrhage. Becroft and Lockett described abundant intra-alveolar siderophages in the lungs of four victims of repeated imposed upper airway obstruction (who also had bleeding from the nose and mouth during these episodes), providing further support for the idea that asphyxia in infants is accompanied by alveolar haemorrhage. ${ }^{6}$ This and other studies have confirmed that substantial numbers of intra-alveolar siderophages are an infrequent feature of uncomplicated SIDS, and should lead to a search for causes of previous alveolar haemorrhage including suffocation. ${ }^{7}$

The paper by Yukawa et al investigates whether acute alveolar haemorrhage is also a marker of accidental and deliberate suffocation post mortem. Alveolar haemorrhage was quantitated in lung sections of 62 consecutive sudden and unexpected infant deaths from the archives of the Department of Forensic Pathology, Sheffield. The authors concede that this is not a population based sample. Six cases were more than a year old. The cases were divided into SIDS and non-SIDS groups on the basis of the original postmortem diagnosis, and then subclassified retrospectively and blind to the results of image analysis. No criteria for this reclassification are given. Haemorrhage was assessed as the percentage of alveolar area occupied by red cells. No attempt was made to correct for inflation. The paper concludes that "if a moderate degree (at least $5 \%$ ) of pulmonary parenchymal haemorrhage is observed, this may be an indicator of airway obstruction for a significant period, either from overlaying, or possibly smothering. The diagnosis of "SIDS" may be being used inappropriately in such cases."

Do the data presented justify excluding babies with more than 5\% haemorrhage from the SIDS group? None of the "typical" SIDS cases in this study showed more than 5\% haemorrhage. However, only three of the original 29 SIDS cases were retrospectively subclassified as "typical" SIDS, and one of these was 12 months old (one might justifiably ask why another two week old baby with $15 \%$ haemorrhage was excluded from the "typical" SIDS group on the basis of age and a probe-patent ductus arteriosus). The number of typical SIDS cases is too small to support an arbitrary limit to the amount of haemorrhage allowed for this diagnosis.

Does alveolar haemorrhage indicate deliberate smothering? Although $45 \%$ of cases in which there was possible or admitted smothering showed more than 5\% haemorrhage, this was not significantly different from SIDS. Ten of the 14 cases ascribed to asphyxia had less than 5\% haemorrhage. More than 5\% haemorrhage was also found in some "atypical" SIDS, co-sleeping, infection, and traumatic deaths. Clearly, more than $5 \%$ alveolar haemorrhage is neither a necessary nor a specific criterion for the diagnosis of deliberate suffocation.

An intriguing finding was that in 11 cases in which overlaying was either admitted consistently or a strong possibility, $73 \%$ showed more than $5 \%$ alveolar haemorrhage, a highly significant difference from non-co-sleeping SIDS cases. Why is pulmonary haemorrhage so prominent among co-sleeping deaths? A glance at the data presented shows that alveolar haemorrhage is a feature of younger SUDI babies, regardless of cause of death. The seven babies under four weeks of age include all four with more than $20 \%$ haemorrhage. From the data presented, the mean percentage area of haemorrhage was $23.7,7.0,2.5$, and 2.36 at 0 to 4 weeks, 4 to 8 weeks, 8 weeks to 3 months, and 3 to 4 months, respectively-a significant difference. Of the 11 overlaying deaths, seven were less than eight weeks old, and four less than four weeks old. This study and others confirm that co-sleeping deaths occur among smaller and younger babies than non-co-sleeping cot 
deaths. ${ }^{8}$ Further work is needed to establish whether it is young age or co-sleeping that is the independent variable accounting for the excess of pulmonary haemorrhage. At the present time it is therefore not justifiable to ascribe co-sleeping deaths to overlaying on the basis of pulmonary haemorrhage.

Yukawa et al are to be congratulated for attempting to bring some objectivity to an area rife with innuendo and conjecture. Fortunately, they show that subjective assessment of alveolar haemorrhage is possible, but tends to underestimate that measured objectively. Their data support their conclusion that the finding of $>5 \%$ alveolar haemorrhage should trigger careful reappraisal of the case. How does a pathologist recognise the cases caused by asphyxia? By looking for other signs of physical abuse, facial petechiae, pressure marks, heavily blood stained fluid in the respiratory tract, staining lung sections for haemosiderin and carrying out a very thorough necropsy, but above all by obtaining a very full history. ${ }^{9-11}$

When everything possible has been done and uncertainty remains, what should we do? Our clear duty is to prevent other infant deaths, and to minimise hurt to others. SIDS was conceived as a registrable cause of death which recognised that infant deaths could be both natural and unexplained. It is a humane and pragmatic diagnosis, and we should not let our personal or research beliefs about the role of head covering, sleeping position, co-sleeping, or overlaying in relation to accidental suffocation prevent us from using it, especially if any perceived lack of care was inadvertent. Only in extreme cases, for example when a demonstrably intoxicated parent may have accidentally overlain their baby, is "unascertained" appropriate. In contrast, if there is well founded concern about deliberate suffocation, based on fact and supported by the literature, then SIDS should not be used because it closes the minds of other health care professionals, and may prevent action by social services in the future. Siblings and future babies may be at risk, and so inaction is not an option. Action must be graduated according to the degree of concern, and might include obtaining further history, sharing concerns with the general practitioner or paediatrician, multidiscipli- nary case review, an investigation by police and social services, or a formal request for review of the case records by a paediatrician experienced in the field. A diagnosis of "unascertained" will generally lead to an inquest where it may be sufficient and acceptable at that stage, and after exhaustive investigation, to explain why the case does not fit the definition of SIDS, that death could have been from natural disease, injury, or accident, and that one was not able to distinguish these possibilities by necropsy examination alone. "Unascertained" is not an accusation, but its indiscriminate use causes distress to parents. It should therefore not be used lightly, nor to cover the pathologist's tail.

Pulmonary haemorrhage is just one factor in many that contributes to making these difficult decisions. It is neither a necessary nor a specific marker of deliberate or accidental suffocation.

P J BERRY

Department of Paediatric Pathology, St Michael's Hospital, Southwell Street, Bristol BS2 8EG, UK

1 Meadow R. Unnatural sudden infant death. Arch Dis Child 1999;80:7-14.

2 Southall DP, Plunkett MCB, Banks MW, et al. Covert video recordings of life-threatening child abuse: lessons for child protection. Pediatrics 1997;100:735-60.

3 Greenough A, Roberton NRC, Rennie JM, et al, eds. Textbook of neonatology, 3rd ed. Part 2. Acute respiratory disease in the newborn. Edinburgh: Churchill Livingstone, 1999:481-607.

4 Montana E, Sorenson WG, Kullman GJ, et al. Acute pulmonary homona E, Sorenson WG, Kullman GJ, et al. Acute pulmonary hemorrhage in infants associated with exposure to Stachy

5 Berry PJ. Pathological findings in SIDS. F Clin Pathol 1992;45(suppl):1116.

6 Becroft DM, Lockett BK. Intra-alveolar pulmonary siderophages in sudden infant death: a marker for previous imposed suffocation. Pathology 1997;29:60-3.

7 Dearborn DG, Infeld MD, Smith PG, et al. Update: pulmonary hemorrhage/hemosiderosis among infants-Cleveland, Ohio, 1993-1996. $M M W R$ 1997;46:33-5.

8 Leach CEA, Blair PS, Fleming PJ, et al. The CESDI study: SIDS infants and potential hazards associated with the sleeping place. Pediatr Pulmonol 1997;24:451.

9 Meadow R. Suffocation, recurrent apnea, and sudden infant death. $\mathcal{F}$ Pediatr 1990;117:351-7.

10 Bamford F, MacFadyen U, Meadow SR, et al. Report of the BPA working party on evaluation of suspected imposed upper airway obstruction. London: Royal Society of Medicine Press, 1994.

11 Green MA. A practical approach to suspicious death in infancy-a personal view. F Clin Pathol 1999;51:561-3. 